\title{
Scanning probe investigations of morphology of new p-type material for organic field-effect transistors
}

\author{
Ovidiu-Gelu Tudose, Sigurd Schrader, Heiko Thiem
}

\section{Introduction}

Organic electronics is a fast growing field of research and technological developments. First applications based on organic electronics have been available on the market for a couple of years, so high resolution colour displays based on organic light-emitting devices are used in digital cameras or mobile phones. Efforts are still going on to find better materials and for a better understanding structure property relationships of organic semiconductors. Although exemplary applications have been realized from polymers and amorphous layers of evaporable molecular materials, crystalline organic semiconductors receive intense attention because the charge-carrier mobilities in these materials are much higher than in polymers or amorphous layers. After optimizing the electronic properties of organic devices (e.g. organic field-effect transistors or organic light emitting diodes) and demonstration of fundamental devices, attention has turned to morphology and composition of the films. Understanding and controlling the morphology of organic films will have a significant impact on the performance of organic-based devices, e.g. by minimizing the number of grain boundaries in the active region of an organic fieldeffect transistor (OFET) [1].

We have investigated with different measuring techniques thin films of 5,5'-Dii(9,9'-di-(butyl)-fluorene)-2,2'bithiophene (DBFBT) prepared on different substrates by high-vacuum evaporation and spin-coating techniques. This material combines thiophene and fluorene moieties, in order to promote either hole or ambipolar transport. Since the latter is usually used for development of complementary logic devices, materials which support ambipolar transport like the fluorene-bithiophene copolymer F8T2 are of great importance for realizing organic complementary logic devices [2]. Such devices are working more stable and are more reliable than other logic devices, which are based on one type of charge carrier transport only. The investigation of DBFBT is, therefore carried out on the background of application in complementary logic devices. Z. Bao et al. [3] showed, that with this class of material hole mobilities of up to $0.1 \mathrm{~cm}^{2} / \mathrm{Vs}$ could be reached, when the morphology of the thin film is optimised by changing substrate temperatures or evaporation rates. They showed an increase in the mobility to 0.06 $\mathrm{cm}^{2} / \mathrm{Vs}$ when the substrate temperature is raised from $25{ }^{\circ} \mathrm{C}$ to $140{ }^{\circ} \mathrm{C}$ during the evaporation process. These high mobilities make this class of material interesting for OFET applications.

\section{Results and discussion}

\subsection{Material}

$5,5^{\prime}$-Di(9,9'-di-(butyl)-fluorene)-2,2'-bithiophene is an organic semiconductor, with the chemical structure shown in fig. 1.

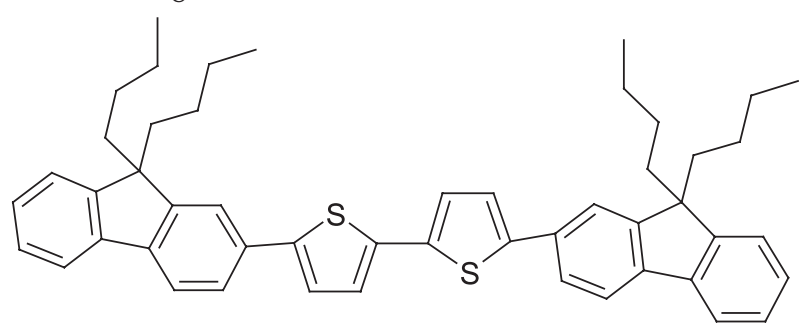

Fig.1. Chemical structure of 5,5'-Di(9,9'-di-(butyl)-fluorene)-2,2'bithiophene (DBFBT).

The thermal properties of DBFBT were determined by a DSC scan. The heating curve shows a melting at $207^{\circ} \mathrm{C}$. Recrystallisation can be detected at $140{ }^{\circ} \mathrm{C}$ in the cooling curve. Thin films of the DBFBT for UV-VIS measurements were prepared on quartz substrates by spin coating from a chloroform solution (5 $\mathrm{mg}$ DBFBT in $10 \mathrm{ml}$ solvent). The UV-VIS absorption and photoluminescence (PL) spectra of the prepared greenish thin films are shown in Fig. 2. The main absorption maximum is at $406 \mathrm{~nm}$. PL spectra of DBFBT thin films show well-resolved maxima at $464 \mathrm{~nm}$ and $590 \mathrm{~nm}$, respectively.

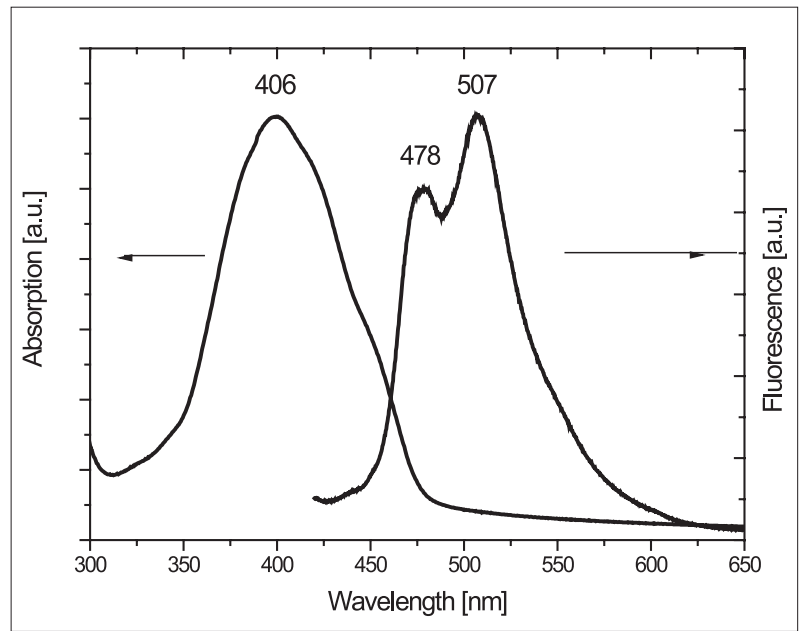

Fig. 2. UV-VIS and photoluminescence spectra of thin films of 5,5'-Di(9,9'-di-(butyl)-fluorene)-2,2'-bithiophene.

Cyclic voltammetry (CV) was used to investigate the oxidation behaviour of the 5,5'-Di(9,9'-di-(butyl)-fluorene)2,2'-bithiophene and to estimate the energetic position of its HOMO level. In Fig. 3 the CV measurement is plotted 
and shows two fully reversible oxidation peaks. Repeated oxidation and reduction has no influence on the redox potential. This is a good proof for the electrochemical stability of the material. The oxidation potential was measured vs. $\mathrm{Ag} / \mathrm{AgNO}_{3}$ as reference electrode at $25^{\circ} \mathrm{C}$ and in $\mathrm{CH}_{2} \mathrm{Cl}_{2}$ as solvent. The calibration of the experiment was made with the standard ferrocene/ferrocenium redox system.

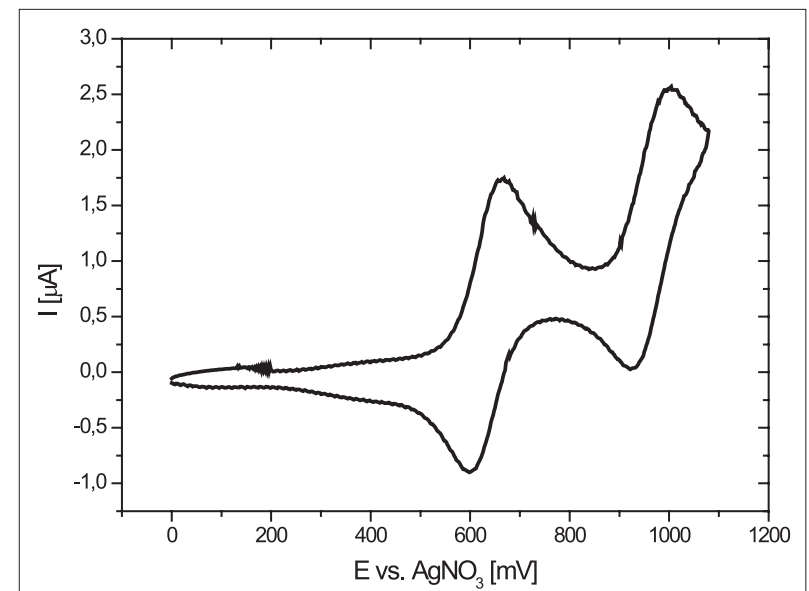

Fig. 3. Cyclic voltammogram of DBFBT in $\mathrm{CH}_{2} \mathrm{Cl}_{2}$ measured with $\mathrm{Ag} / \mathrm{AgNO}_{3}$ as reference electrode.

Taking - $4.8 \mathrm{eV}$ as the HOMO level of the ferrocene system, the HOMO level of the material is $-5.3 \mathrm{eV}$ [4]. With this value a LUMO level of $-2.6 \mathrm{eV}$ can be calculated with an optical band gap of $2.7 \mathrm{eV}$ taken from the absorption edge of $475 \mathrm{~nm}$ (see fig. 2).

\subsection{AFM results for DBFBT thin films}

Thin films of DBFBT were prepared using different techniques: high-vacuum evaporation and spin coating. Firstly high vacuum evaporated DBFBT thin films were prepared on various substrates: silicon wafers and glass slides coated with indium tin oxide (ITO). Films were deposited by thermal evaporation in a diffusion-pumped vacuum system with a typically base pressure of less than $8 \times 10^{-6}$ Torr. The substrates were held at room temperature and the DBFBT was deposited with $1 \AA /$ s, with a final average thickness of $150 \mathrm{~nm}$. The temperature of the sample holder was controlled at a large temperature range by resistive heating elements and water-cooling. The temperature of the substrate was monitored by iron-constantan thermocouples. A quartz oscillator monitored the deposition rate. Also, during the deposition of thin films, the deposition rate was kept constant. A shutter allowed adjusting the evaporation rate to the desired value before the deposition of the DBFBT thin film was performed. The material was loaded to a crucible, which was resistively heated (here the material was heated up to $240^{\circ} \mathrm{C}$ ).

Second, spin-coated DBFBT thin films were also prepared on different substrates. The spin-coating technique involves the application of a liquid drop of diluted layer material to the centre of the fast spinning substrate, where it is spread towards the whole surface. Usually two parameters influence mainly the thickness, but also other properties of the thin films: spin-speed and viscosity. Different spin-speeds (2000 rpm, $1500 \mathrm{rpm}, 1000$ rpm) were used and the viscosity was kept at a constant value. A concentration of $5 \mathrm{mg}$ material/10ml chloroform has been used to prepare the thin films. The range of film thickness achieved by spin coating is $100-150 \mathrm{~nm}$, dependent on the spin speed.

The film morphology was characterized using an Atomic Force Microscope from Park Scientific Instruments, operating in non-contact mode.
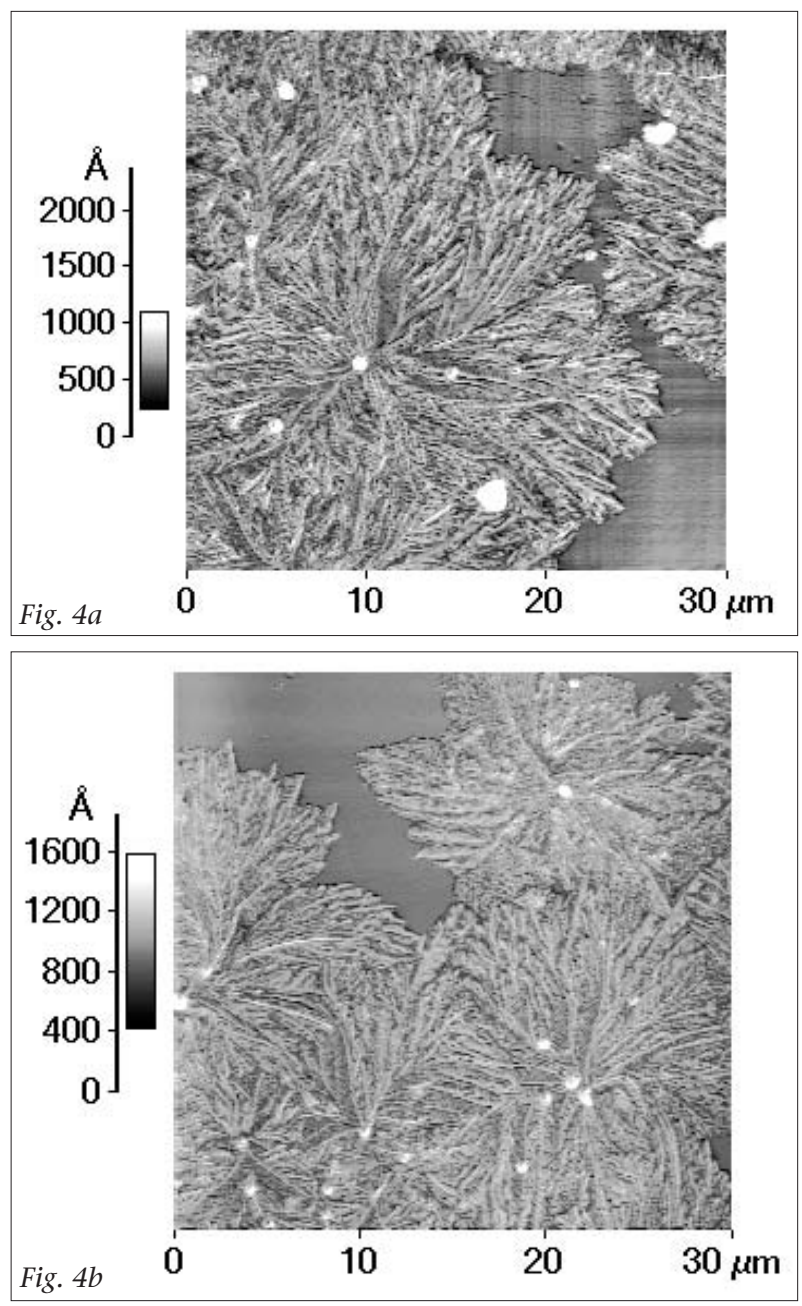

Fig. 4: $30 \times 30 \mu^{2}$ atomic force microscope image of DBFBT layer with average thickness of $150 \mathrm{~nm}$ deposited by high vacuum evaporation technique on ITO substrate (a) and on silicon substrate (b).

Atomic force microscopy (AFM) images of the topology of DBFBT films deposited by high-vacuum evaporation technique are shown in fig. 4 . The $30 \times 30 \mu \mathrm{m}^{2}$ scan shows nucleated islands grown laterally by capturing diffusing molecules. Diffusive capture results in morphology determined by 'hit and stick' mechanism basis of diffusionlimited aggregation. In some systems, which are described with diffusion-limited aggregation (DLA) mechanism, the elementary units that successively stick to form the aggregate remain thereafter at relatively fixed positions, thus causing an intrinsic rigidity in the structure. The statistical properties characterizing the disorder of the particle position are quenched by the growth process itself. This is an important limitation that makes it impossible to apply a diffusion-limited model to describe aggregating systems, like the ones involving rearrangement within the clusters [5] or systems of polymer chains in solution [6], that consists of elements of a fluctuating nature. A 
realistic model for describing growth in this class of systems must include information not only about the colliding events that lead to the irreversible build up of the structure, but also about the equilibrium configurations that result from the Brownian motion of the internally articulated constituents.

Aggregation takes place when identical particles are joined into clusters according to some rule: the statistical properties characterizing the positional disorder are a combination of quenching and annealing processes but more crucially, the consecutive attachments of the incoming diffusive particles will now be biased by the fluctuations of the existing aggregate. Aggregation almost always leads to ramified structures with fractal geometry. The process starts with a single seed particle at the origin. A particle is then released from a distant point and is allowed to undergo a diffusive motion until it arrives at a site adjacent to the seed, where it sticks permanently to the seed. Additional particles are released one by one and are attached to the growing aggregate in the same way $[7,8]$.

Atomic force microscopy (AFM) images of the topology of the DBFBT films deposited by spin-coating technique are shown in fig. 5.
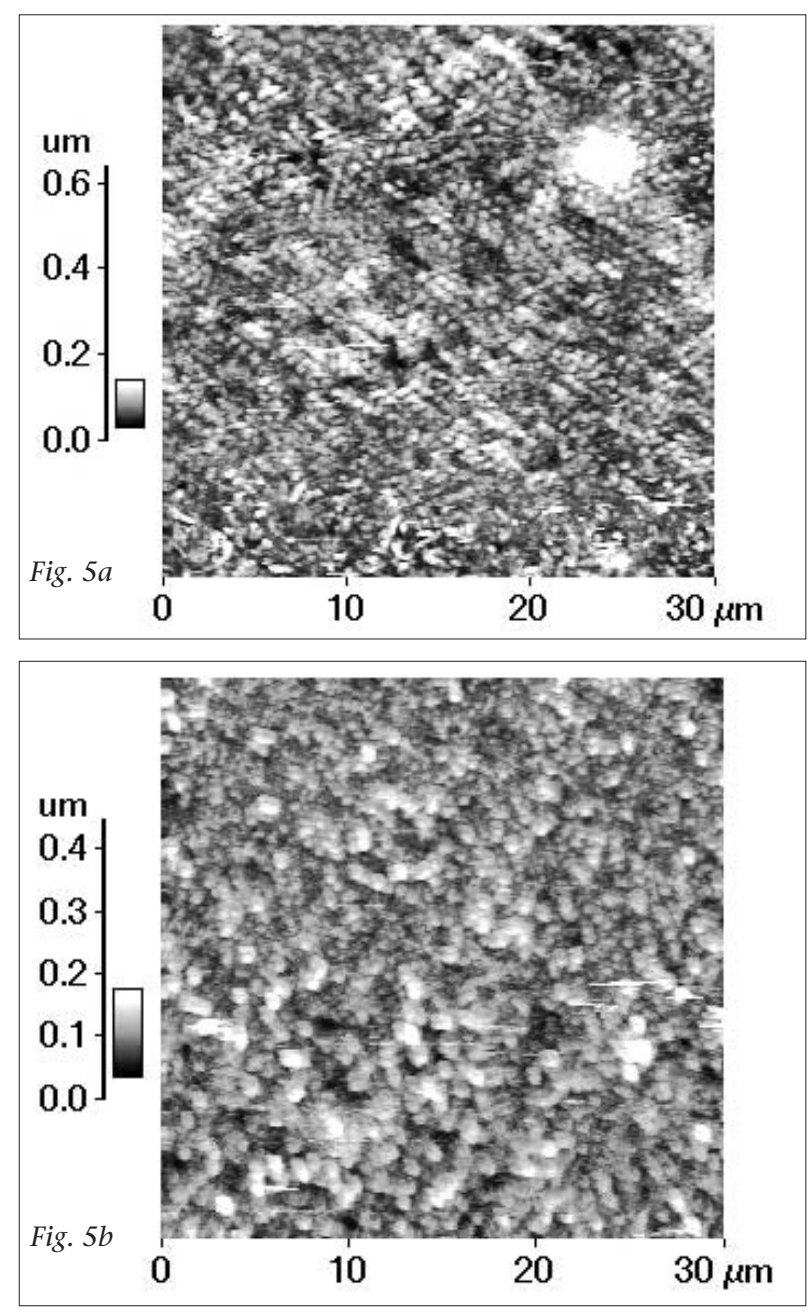

Fig. 5: $30 \times 30 \mu^{2}$ atomic force microscope image of 5,5'-Di(9,9'-di(butyl)-fluorene)-2,2'-bithiophene layer with a thickness of $150 \mathrm{~nm}$ (a) and $100 \mathrm{~nm}$ (b) deposited on ITO substrate by spin-coating.
According to the application requirements, the thickness of the DBFBT thin films prepared by spin coating can be adjusted. Depending on the spin-speed, coating thickness can be varied, e.g., from $100 \mathrm{~nm}$ to $150 \mathrm{~nm}$, with one single spin coating step. Results obtained from $150 \mathrm{~nm}$ and $100 \mathrm{~nm}$ thick coating layers are shown in Figs. 5(a) and 5(b). These figures are 2D pictures of an area of 30 x $30 \mu \mathrm{m}^{2}$ on the coating surface, which shows an average surface roughness RMS of $27 \mathrm{~nm}$ (fig. 5(a)) and 24 $\mathrm{nm}$ (fig. 5(b), respectively). The DBFBT thin films show a microcrystalline structure, as it can be seen from the above figures.

\section{Conclusions}

The structure and morphology of thermally evaporated and spin-coated DBFBT films have been studied. In the preparation step of thermally evaporation high-vacuum thin films, indications for a mechanism based on diffusion-limited aggregation (DLA) were detected. By comparing the AFM pictures of DBFBT deposited by these two methods, it was demonstrated that the deposition technique influences the topography of the thin films too. From the high-vacuum thermally evaporation method well ordered structures, and from spin-coating a micro-crystalline structures are obtained. On the other hand, in case of thermally evaporated material in high-vacuum deposition, DBFBT films deposited on different substrates (silicon and ITO) did not show different morphologies. Fractal growth by the mechanism based on diffusion-limited aggregation has been detected on different substrates.

In order to apply DBFBT thin films further studies are necessary including $\mathrm{x}$-ray investigations, in order to get information about the unit cell, angle resolved ultraviolet photoelectron spectroscopy measurements for obtaining the valence electronic structure of DBFBT and time-of-flight measurements to obtain information about transport properties. Here initial information for all these studies is provided because the mentioned properties depend all on the actual morphology as described in the present paper.

\section{Acknowledgements}

The authors would like to thank Dr. Peter W. Schmidt (University of Applied Sciences Wildau) for critical reading of the manuscript, to Monika Ehlert (University of Potsdam) for the help with DSC measurements, to Burkhard Stiller (University of Potsdam) for the help with AFM measurements and Prof. Peter Strohriegl (University of Bayreuth) for many fruitful discussions. Financioa support of the European Commission under contract number HPRN-CT-2002-00327 (RTN-project EUROFET) is gratefully acknowledged. 


\section{Bibliography}

[1] D.J. Gundlach, Y.Y. Lin, S.F. Nelson, D.G. Schlom and T.N. Jackson, IEEE Electron Device Lett. 18, 87 (1996).

[2] L.-L. Chua, J. Zaumseil, J.-F. Chang, E. C.-W. Ou, P. K.-H. Ho, H. Sirringhaus, R. H. Friend, Nature 434, 194 (2005).

[3] H. Meng, J. Zheng, A.J. Lovinger, B.-C. Wang, P. G. Van Patten, Z. Bao, Chem. Mater. 15, 1778 (2003).

[4] J. Pommerehne, H. Vestweber, W. Guss, R. F. Mark, H. Bässler, M. Porsch, J. Daub, Adv. Mater. 7, 55 (1995).

[5] D.W. Schaefer, J.E. Martin et. al., Phys. Rev. Lett. 52, 2371 (1984).

[6] M.R. Gittings, L. Cipelletti, V. Trappe, D.A. Weitz, M., J. Phys. Chem. B 104, 4381 (2000).

[7] C.I. Mendoza, C.M. Marques, Physica A 335, 305 (2004).

[8] B.B. Mandelbrot, B. Kol and A. Aharony, Phys. Rev. Lett. 88, 55501-1-4 (2002).

\section{Authors}

Dipl. Phys. Ovidiu-Gelu Tudose

University of Applied Sciences Wildau

Friedrich-Engel-Str. 63, 15745 Wildau, Germany

Tel. +49 3375 508-280

tudose@igw.tfh-wildau.de

Prof. Dr. rer. nat. habil. Sigurd Schrader

University of Applied Sciences Wildau

Friedrich-Engel-Str. 63, 15745 Wildau, Germany

Tel. +49 3375 508-293

schrader@igw.tfh-wildau.de

Dipl. Chem. Heiko Thiem

University of Bayreuth, Macromolecular Chemistry I Universitätsstr. 30, NW II, 95440 Bayreuth, Germany heiko.thiem@uni-bayreuth.de 